\title{
Erratum to: Ten Years' Experience in Robotic-Assisted Thoracic Surgery for Early Stage Lung Cancer
}

\author{
Monica Casiraghi ${ }^{1}$ Domenico Galetta ${ }^{1}$ Alessandro Borri ${ }^{1}$ Adele Tessitore ${ }^{1}$ Rosalia Romano ${ }^{1}$ \\ Cristina Diotti $^{1}$ Daniela Brambilla ${ }^{1}$ Patrick Maisonneuve ${ }^{2}$ Lorenzo Spaggiari ${ }^{1,3}$
}
${ }^{1}$ Department of Thoracic Surgery, IEO, European Institute of Oncology IRCCS, Milan, Italy
2 Department of Epidemiology and Biostatistics, IEO, European Institute of Oncology IRCCS, Milan, Italy
${ }^{3}$ University of Milan, Department of Oncology and Hemato-oncology,
Milan, Italy

Thorac Cardiovasc Surg

Address for correspondence Monica Casiraghi, MD, Department of Thoracic Surgery, IEO, European Institute of Oncology IRCCS, Via Ripamonti 435, Milano 20141, Italy (e-mail: monica.casiraghi@ieo.it).

\section{ERRATUM}

The authors have informed the Editor-in-Chief on August 22, 2019, that the first and second affiliations were not given correctly in their article published online on April 1, 2018. The doi of the original article is: $10.1055 / \mathrm{s}-0038-1639575$.

Corrected affiliation should read:

${ }^{1}$ Department of Thoracic Surgery, IEO, European Institute of Oncology IRCCS, Milan, Italy ${ }^{2}$ Department of Epidemiology and Biostatistics, IEO, European Institute of Oncology IRCCS, Milan, Italy

Affiliations have been corrected in the article. 